\title{
Pobreza extrema de mujeres indígenas en México. Estudio de caso para la Zona Maya de Quintana Roo
}

\author{
Extreme poverty in indigenous women in Mexico. Case study for Quintana Roo Maya Zone
}

Miguel Ángel Barrera Rojas ${ }^{1}$

\section{Resumen}

Esta investigación ha analizado la pobreza en la población indígena de la Zona Maya del Estado de Quintana Roo en México. En trabajo de campo se recopiló información sobre el ingreso corriente en los hogares de las localidades de estudio para posteriormente aislar a los individuos varones y centrar el análisis en la población femenina. Como método de contraste para cuantificar la pobreza extrema se utilizó la metodología propuesta por el Banco Mundial (línea de pobreza extrema) y del Consejo Nacional de Evaluación de la Política de Desarrollo Social (líneas de bienestar mínimo y de bienestar). Los resultados se presentan en gráficos donde se trazaron las líneas de pobreza tanto del Banco Mundial como del Consejo Nacional para la Evaluación para contrastarlos con el Ingreso Corriente de cada mujer de la muestra.

Palabras clave: Pobreza extrema, Zona Maya, Política social, Indígenas, Mujeres

\section{Abstract}

This investigation has analyzed the poverty in the indigenous population from the Mayan Zone in Quintana Roo State in Mexico. In the work field, it was compiled information about the regular incomes in homes from the places in study to separate men afterwards and center the analysis in the feminine population. With the contrast method to quantify the extreme poverty the proposed methodologies (extreme poverty line) by the World Bank and the Evaluation National Council of the Social Development Policy (minimum wellbeing and wellbeing lines) were used. The results show in graphics where the poverty lines for the World Bank as the Evaluation National Council of the Social Development Policy to contrast them with the Regular Income of each woman in the sample.

Keywords: Extreme poverty, Mayan Zone, Social Policy, Indigenous, Women.

1 Doctor en Geografía por la Universidad de Quintana Roo. Profesor de la División de Ciencias Políticas y Humanidades de la Universidad de Quintana Roo. Correo: miguel.barrera@uqroo.edu.mx; No. ORCID: https://orcid.org/oooo-ooo2-9240-7646

Recibido: 05/02/2018 Aprobado: 30/04/2018 


\section{Introducción}

En la literatura sobre pobreza son bastos los trabajos que se enfocan en este problema desde la perspectiva urbana, rural, y de manera agregada en cuanto al género; sin embargo, son muy pocos los esfuerzos académicos que se avocan a analizar la situación de pobreza en mujeres indígenas. Parte de esta escasez se debe a que los datos disponibles por parte de los gobiernos se quedan a nivel estatal o municipal. De ahí que este texto cobre relevancia pues la metodología arroja datos a nivel localidad sobre el fenómeno de la pobreza.

Si bien el gobierno mexicano a través del Consejo Nacional de Evaluación de la Política de Desarrollo Social (CONEVAL) y de la Secretaría de Desarrollo Social (SEDESOL) han hecho importantes estudios sobre el comportamiento de la pobreza, también es cierto que no existen trabajos que hagan medición de la pobreza a un nivel tan desagregado para la zona Maya de Quintana Roo como el que se ofrece en este esfuerzo académico. En este sentido, el objetivo que se plantea es medir el nivel de pobreza en que viven las mujeres de la zona de estudio. Para el cumplimiento de dicho objetivo se recurrió al uso de la línea de pobreza extrema de Banco Mundial y de las líneas de Bienestar y Bienestar Mínimo del CONEVAL. De igual modo se reconstruyó la variable ingreso corriente para compararlo con las líneas arriba mencionadas.

\section{Revisión de la literatura}

En la década de 1980 la política de desarrollo social en México tuvo un giro sumamente marcado. Pasó el Estado mexicano de ser benefactor a sumergirse de lleno en el neoliberalismo bajo las directrices de organismos internacionales como el Fondo Monetario Internacional y el Banco Mundial. Como parte de estas directrices hubo (y continúa) una drástica reducción del gasto público dirigido a salud, educación, cultural y ciencia, lo que derivó en incrementos de las tasas de desempleo, en caída de las tasas de crecimiento, mismas que incrementaron las tasas de pobreza en México (Zepeda, 1994 Sen, 1998; Escalante, 2015). Como respuesta a las rampantes tasas de pobreza el gobierno federal, desde la presidencia de Ernesto Zedillo, diseñó una serie de políticas públicas de combate a la pobreza donde el componente principal fueron las transferencias monetarias. Este tipo de estímulos, para algunos autores como Esquivel (2009, 2015) no terminan de resolver el problema de la pobreza, incluso, resultan hasta perjudiciales porque la carga fiscal hacia las personas pobres se ha incrementado; mientras que para instancias como la CEPAL (1990, 1996, 2003, 2014) e incluso el BM (1992, 2001,2004, 2013) las colocan como la esperanza más confiable para reducir la brecha de pobreza.

La pobreza se puede explicar desde diferentes enfoques, uno de los más importantes es de Ruggeri, Saith y Stewart (2003), quienes plantean que la pobreza se debe a la imposibilidad de tener ingreso o de hacer consumo, todo esto en términos de líneas de 
pobreza. Para la CEPAL (2004), si bien la pobreza es más una cuestión multifactorial, también es definida como una condición de infra consumo y de vulnerabilidad en el ingreso. Ambas definiciones son muy generales y asumen que la pobreza es igual de intensa para hombres, mujeres, niños, ancianos o indígenas, cosa que no es así. La misma CEPAL (2004) reporta que fue en la década de 1980 cuando se iniciaron los primeros esfuerzos por abordar la pobreza desde la perspectiva de género, las primeras investigaciones al respecto "Identificaron una serie de fenómenos dentro de la pobreza que afectaban de manera específica a las mujeres y señalaron que la cantidad de mujeres pobres era mayor a la de los hombres, que la pobreza de las mujeres era más aguda que la de los hombres y que existía una tendencia a un aumento más marcado de la pobreza femenina" (p. 12), esto ha derivado en esfuerzos académicos para indagar en este problema. Y, como parte de dichos esfuerzos se planteó este trabajo para evidenciar la intensidad de la pobreza en el contexto indígena mexicano. Para ello se eligió hacer trabajo de campo en la región de estudio elegida:

La Zona Maya de Quintana Roo, México. Esta región está formada por los dos municipios con mayor número de población indígena de la etnia maya y de monolingües mayas (yucateco) del estado de Quintana Roo. Las localidades de estudio son José María Morelos, Dos Aguadas, El Naranjal, San Carlos, San Diego, Dziuché, en el municipio de José María Morelos; y, Felipe Carrillo Puerto y Noh-Bec, en el municipio de Felipe Carrillo Puerto.

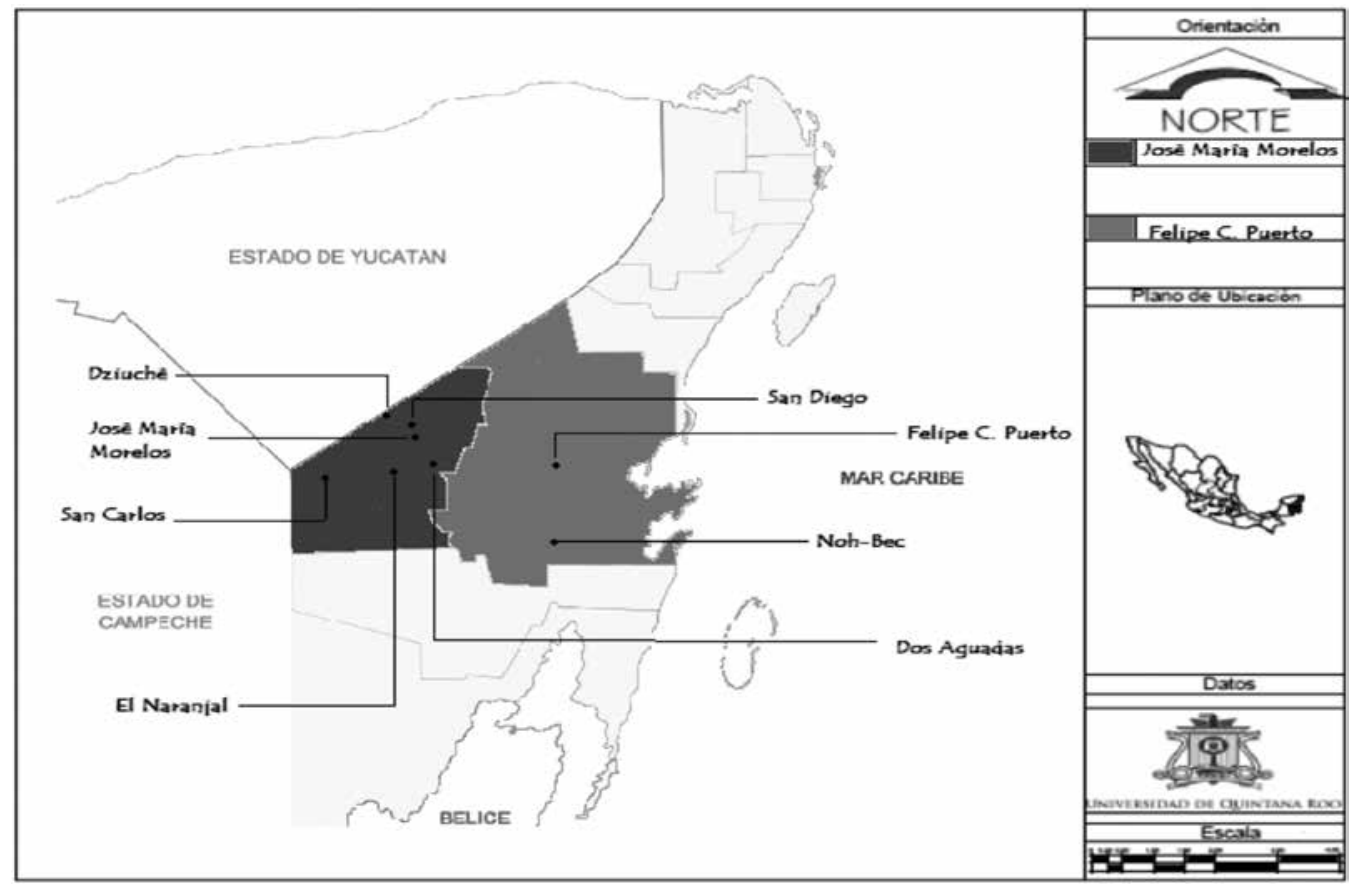

Figura 8: Mapa de Región y localidad de estudio. 
Dentro de los informes más recientes sobre pobreza en el mundo se señala que:

En 2014, por cada 100 hombres viviendo en hogares pobres en la región, habían 118 mujeres en similar situación, esto evidencia la falta de autonomía económica de las mujeres, quienes en ausencia de otros ingresos del hogar son más proclives a estar en situación de pobreza, situación que se agudiza en hogares con mayor presencia de niños y niñas (CEPAL, 2016).

Esto refleja que las políticas de combate a la pobreza no han surtido el mismo efecto en hombres y mujeres, dejando a estas últimas en una situación sumamente vulnerable (Bareiro, 1994; Bravo, 1998, 2004; CEPAL, 2014), en palabras de Sen (1998) se debe analizar la pobreza en que viven las mujeres pues esta condición las torna vulnerables, dependientes del ingreso de los hombres y de los programas de Transferencias Monetarias Condicionadas.

México, como país miembro de la Organización de las Naciones Unidas se comprometió en el 2000 a cumplir los Objetivos del Milenio, entre los que destaca el número "1.A. Reducir a la mitad, entre 1990 y 2015, la proporción de personas con ingresos inferiores a 1 dólar por día" (ONU, 2000, sin página). Esta medida de 1 dólar norteamericano por día surgió en 1991, con el fin de ecualizar el poder adquisitivo de los ingresos, la moneda local se expresa en términos de su equivalencia de poder adquisitivo mediante el uso de dólares de paridad del poder adquisitivo (PPA). En 1991 se estimó una línea internacional de pobreza equivalente a 1 US\$ PPA diario a precios de 1985 , razón por lo cual la línea se dio a conocer como 1 dólar al día. En el 2000 la línea fue recalculada a precios de 1993, y se fijó en 1,08 US\$PPA. Posteriormente, sobre la base de los nuevos índices de PPA generados por el Programa de Comparación Internacional de 2005, se determinó un nuevo umbral, de 1,25 US\$PPA diario (CEPAL, 2016).

En la literatura existente sobre la pobreza extrema, el énfasis se concentra en la distribución del ingreso, pues éste determina si el individuo está encima o debajo del indicador en cuestión. Al respecto Feres y Mancero (2001), establecieron una división sobre las metodologías para la medición de la distribución del ingreso muy práctica y que se ajusta a la realidad latinoamericana. En su estudio, estos autores, plantean primero la idea de diferenciar los indicadores de bienestar, al respecto plantean que "La identificación de los pobres implica realizar una comparación entre distintos niveles de bienestar. Por tanto, se debe elegir una variable cuantificable que actúe como indicador del nivel de bienestar de las personas" (Feres \& Mancero, 2011, p. 50). Por lo anterior es que el Ingreso Total es la variable que se ha elegido como cuantificable y las líneas de pobreza del Banco Mundial y CONEVAL como método de contraste. Este método constituirá un auxiliar teórico metodológico fundamental, ya que el ingreso corriente per cápita se contrastará contra la línea de bienestar y bienestar mínimo que el CONEVAL establece para México y contra las líneas de pobreza extrema del Banco Mundial. Es importante señalar que el origen de este método se remonta a 1880 
cuando en una conferencia de la Royal Statistical Society se propuso segmentar por líneas a la población en términos de su ingreso, es decir, las familias que cumplieran con cierta cantidad de ingreso y de nivel de empleo se encontraban "por encima de la línea de pobreza" (Gillie, 1996: 715). Su uso académico ha sido de tal tamaño que incluso organismos internacionales como el Banco Mundial (BM) lo utilizó en sus informes de 1992, 2001, 2004 y 2013 para definir como pobreza extrema a la población que vive al día con menos de $\$ 1.25$ y de $\$ 2.50$ dólares americanos de paridad de poder adquisitivo (PPA).

Actualmente el propio Consejo Nacional de Evaluación de la Política de Desarrollo Social (CONEVAL) se encarga de medir la pobreza en México de manera multidimensional, siendo el Ingreso corriente per cápita, el rezago educativo promedio en el hogar, el acceso a los servicios de salud, el acceso a seguridad social, la calidad y espacios de la vivienda, el acceso a los servicios básicos en la vivienda, el acceso a la alimentación y el grado de cohesión social en cuanto a las dimensiones a evaluar. En lo que se refiere al Ingreso corriente el CONEVAL $(2010,2014)$ señala que "se identifica a la población en situación de pobreza como aquella que padece al menos una carencia social y tiene un ingreso inferior al valor de la línea de bienestar" (p. 8). De ahí que se haya elegido utilizar estas líneas propuestas para el caso de las comunidades de estudio de esta tesis doctoral. Para el caso concreto de nuestro país, según el CONEVAL (2010; 2014):

En México se han empleado metodologías similares, pero con valores de líneas distintos, los cuales resultan de la construcción de canastas con diferentes composiciones; como el caso de la línea de pobreza elaborada por la Coordinación General del Plan de Zonas Deprimidas y Grupos Marginados (COPLAMAR) en los años setenta y la propuesta de líneas de pobreza por parte del Comité Técnico para la Medición de la Pobreza (CTMP) en el año 2002 (p. 8).

Estas mediciones de la pobreza en México han arrojado dos realidades de México: el México urbano y el México rural (Cornelius \& Myhre, 1998). Esta última realidad igual se puede dividir en dos: lo rural y lo rural indígena (Delgado, 1999; Esquivel, 2009). Y es precisamente en esta última realidad donde se sitúa el presente texto, en la zona indígena Maya de Quintana Roo, esta zona se compone principalmente de los municipios de Felipe Carrillo Puerto y José María Morelos. Ambos municipios tienen vocación productiva que tiende al campesinado y en menor medida a la prestación de servicios de turismo alternativo y comercio. Dicha vocación provee un contexto donde el desempleo esta una constante, las opciones laborales son pocas, la descampesinización es latente y la ausencia de servicios básicos es evidente (Ramírez \& Ibarra, 2015; Jessoe, Manning y Taylor, 2016; Gaxiola y Castro, 2017). Así el objetivo de este trabajo es indagar el número de mujeres, pues son ellas las más vulnerables en términos del ingreso, que viven por debajo de los parámetros de pobreza extrema y bienestar mínimo que establecen Banco Mundial y el CONEVAL respectivamente. 


\section{Materiales y métodos}

Para dar respuesta al objetivo de este trabajo se construyó la variable Ingreso Corriente (IC) de la misma manera en que el Instituto Nacional de Estadística Geografía e Informática (INEGI) lo hace para la Encuesta Nacional de Ingreso y Gasto de los Hogares (ENIGH). Dicho IC "Se forma por las entradas monetarias y no monetarias que satisfacen estos tres criterios: Regularidad, Disponibilidad, Patrimonio" (INEGI, 2013: 1). Este IC, está dividido en cinco partes: 1) Ingreso por la vía del trabajo (IT); 2) Renta de la propiedad (IR); 3) Transferencias (TR); 4) Estimación del alquiler de la vivienda (AV); y 5) Otros ingresos corrientes (OI):

$$
I C=I T+I R+T R+A V+O I
$$

Para IT se consideran todos aquellos ingresos que sean por medio el trabajo subordinado, trabajo independiente u otras formas de empleo. IR representa dos tipos de ingresos, el primero es la participación monetaria que los integrantes del hogar tienen proveniente de cooperativas y sociedades de empresas; la segunda es como ingreso por el arrendamiento de activos fijos dentro y fuera del país, así como los dividendos de la posesión de activos financieros y cuentas de ahorro. El rubro de TR es definido por INEGI (2013) como "las entradas monetarias recibidas por los integrantes del hogar y por las cuales el proveedor o donante no demanda retribución de ninguna naturaleza" (p. 4). En lo que refiere a AV se pregunta por el monto que se paga por el alquiler de la vivienda o bien una estimación, en caso de vivir en casa propia, de lo que se pagaría por una vivienda en alquiler con las mismas características de la que se tiene. OI representa ingresos que estén fuera de los rubros anteriormente descritos.

La muestra que se calculó fue igual a 140 hogares, sin embargo, la recolecta de información acumuló un total de 196 hogares, mismos que agruparon un total de 911 individuos, y se determinó de la siguiente manera:

$$
n=z_{\propto}^{2}\left(\frac{N * p * q}{i^{2}(N-1)+z_{\propto}^{2} * p * q}\right)
$$

Donde: $N \rightarrow$ tamaño de la población $\left(10,182^{2}\right) ; z \rightarrow$ valor de la distribución de Gauss $=2.38$ para $98 \%$ de intervalo de confianza; $p \rightarrow$ Prevalencia esperada del parámetro a evaluar (0.5); $q \rightarrow 1-p=0.5 ; i \rightarrow$ error que se prevé cometer (0.10). Para la afirmación anterior se utilizó la Penn World Table se determinó que el tipo de cambio del dólar a precios de 2005 es de $\$ 10.897^{3}$.

2 De acuerdo al censo del INEGI (2010) el número de hogares en las localidades de estudio es El Naranjal (135), San Carlos (27), San Diego (120), Dziuche (702), José María Morelos (2626), Dos Aguadas (40), Noh-Bec (505) y Felipe Carrillo Puerto (6027).

3 Si considerásemos el tipo de cambio promedio de marzo 2016 (\$18.50) entonces el porcentaje de hogares que viven con menos de 1.25 dólares americanos diarios sería del $38 \%$. 
Cuadro 14: Penn World. Fuente: University of California Davis

\begin{tabular}{|c|c|c|c|}
\hline Year & Mexico / Exchange Rate (US=1) & $\begin{array}{c}\text { Mexico / National Currency (Local } \\
\text { Currency Unit) (N/A) }\end{array}$ & $\begin{array}{c}\text { Mexico / Purchasing Power } \\
\text { Parity over GDP (US=1) }\end{array}$ \\
\hline 2005 & 10.8978916700 & "Mexican Peso" & 7.4652324510 \\
\hline
\end{tabular}

\section{Resultados y discusión}

El trabajo de campo que se realizó arrojó una muestra de 911 personas. De las cuales 462 fueron hombres (50.71\%) y 449 mujeres (49.28\%). De estas, 289 personas (31.72\%) reportaron ser mayaparlantes natos, mientras que 549 personas $(60.26 \%)$ indicaron que su lengua materna es el castellano, y finalmente, un dato que llamó la atención fue que 73 personas (8.01\%) tienen por lengua materna la Maya Tzotzil, que es una lengua típicamente hablada en Guatemala y Chiapas. El promedio de años que la muestra asistió a la escuela es de 6.64, en los hombres es de 6.54 y de las mujeres es de 6.70 es decir, tienen la primaria concluida e iniciado el primero de secundaria, el tamaño medio del hogar de la muestra es de 4.3 miembros.

En lo que refiere al IC, en el cuadro 2 se muestra la estadística básica de dicha variable y se observa que el dato con ingreso más alto es superior al millón de pesos anual y es hombre, el ingreso máximo reportado por una mujer es de casi una cuarta parte del dato máximo general. Otra peculiaridad de estos datos radica en que los hombres con ingreso igual a cero son 135 (29.22\% del total de los hombres), mientras que las mujeres suman 190 (42.31\% del total de las mujeres). De ahí la insistencia de centrar este artículo a la pobreza extrema entre las mujeres.

Cuadro 15: Estadística básica del ingreso corriente

\begin{tabular}{|l|l|l|l|}
\hline & \multicolumn{1}{|c|}{ Total } & \multicolumn{1}{c|}{ Hombres } & \multicolumn{1}{c|}{ Mujeres } \\
\hline Media & $\$ 19,031.13$ & $\$ 25,004.53$ & $\$ 12,877.42$ \\
\hline Mediana & $\$ 4,800.00$ & $\$ 10,000$ & $\$ 4,500.00$ \\
\hline Moda & $\$ 0$ & $\$ 0$ & $\$ 0$ \\
\hline Máximo & $\$ 1,080,000.00$ & $\$ 1,080,000.00$ & $\$ 255,000$ \\
\hline Mínimo & $\$ 0$ & $\$ 0$ & $\$ 0$ \\
\hline Desviación estándar & $\$ 58,319.12$ & $\$ 62,011.79$ & $\$ 26,272.18$ \\
\hline
\end{tabular}

En la figura 2 se observa el ingreso de toda la muestra, resalta en ella tanto el outlayer máximo, cuyo IC es superior al millón de pesos anuales, como los outlayer mínimos

4 Más información en http://cid.econ.ucdavis.edu/pwt.html. Los datos reflejan el año 2015 pues su recolección fue a lo largo del 2014. 
que están con IC igual a cero. La figura 3 establece una comparación entre los ingresos de hombres y mujeres, y es claro en el gráfico la desigualdad y vulnerabilidad que parecen las mujeres, pues son ellas las que mayor incidencia tienen de IC igual a cero y la mujer que mayor ingreso registra no es ni cercana al hombre con mayor ingreso. De ahí el énfasis de este trabajo en esbozar el fenómeno de la pobreza extrema en mujeres indígenas de Quintana Roo, México.

La figura 4 muestra el Ingreso corriente de las mujeres de la muestra por localidad ilustra con claridad que los mayores ingresos se concentran en aquellas localidades que son menos rurales, eso obedece a que, aunque escasa, la disponibilidad de empleo fuera del ámbito agrícola existe, sin embargo, ello no les exime de la dura situación de pobreza que se vive en dicha región y que se expone en las siguientes figuras.

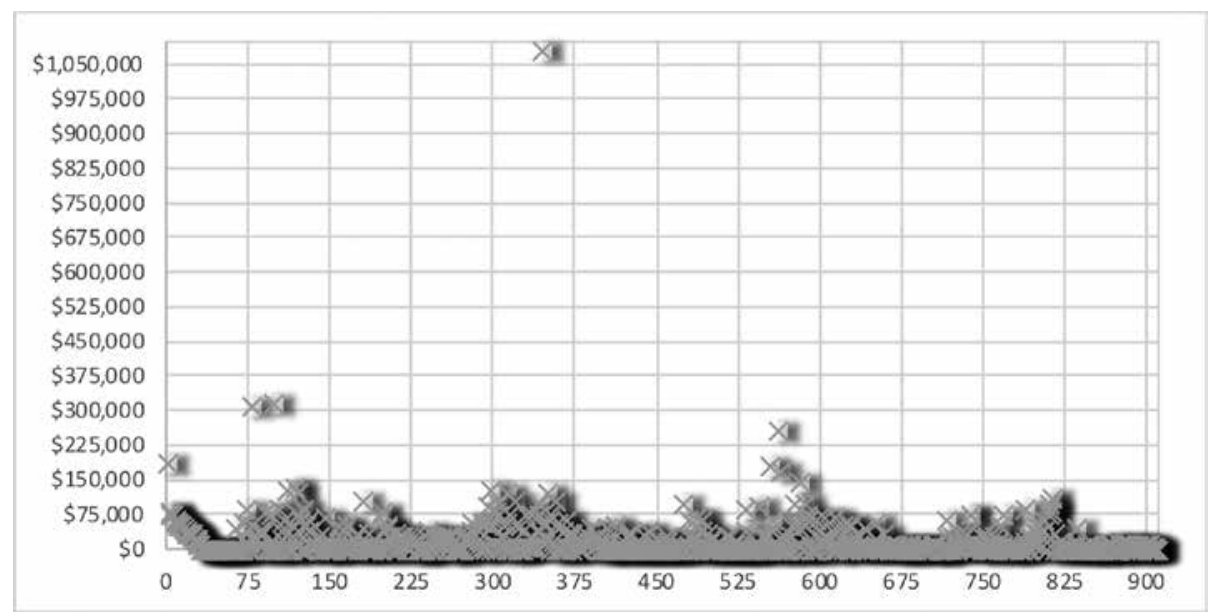

Figura 9: Ingreso corriente para toda la muestra.

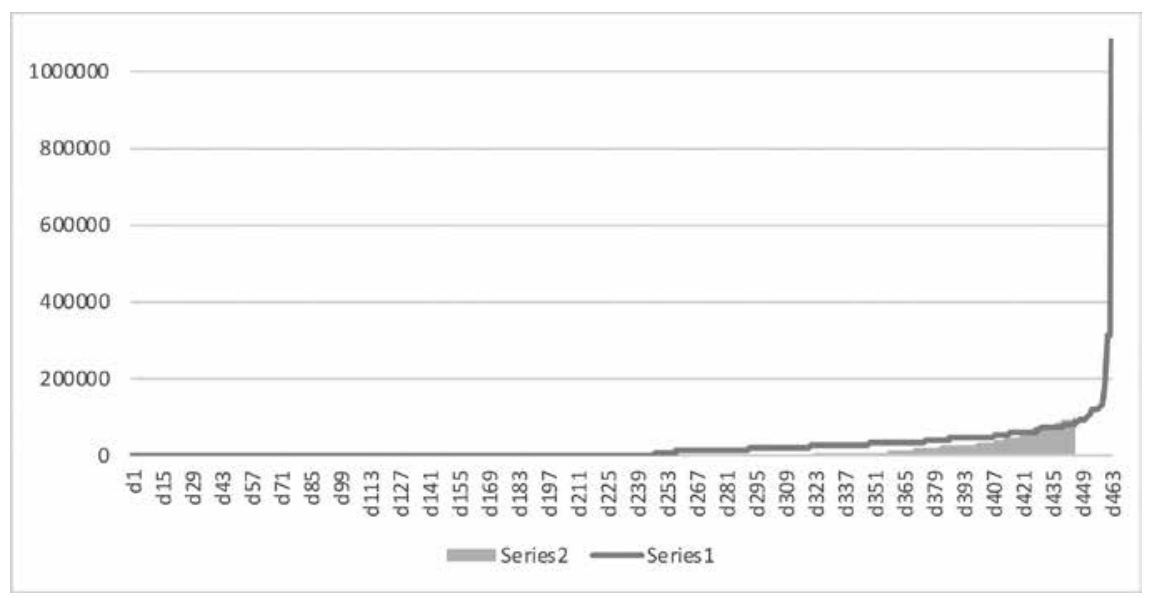

Figura 10: Ingreso corriente de hombres y mujeres en la muestra. 


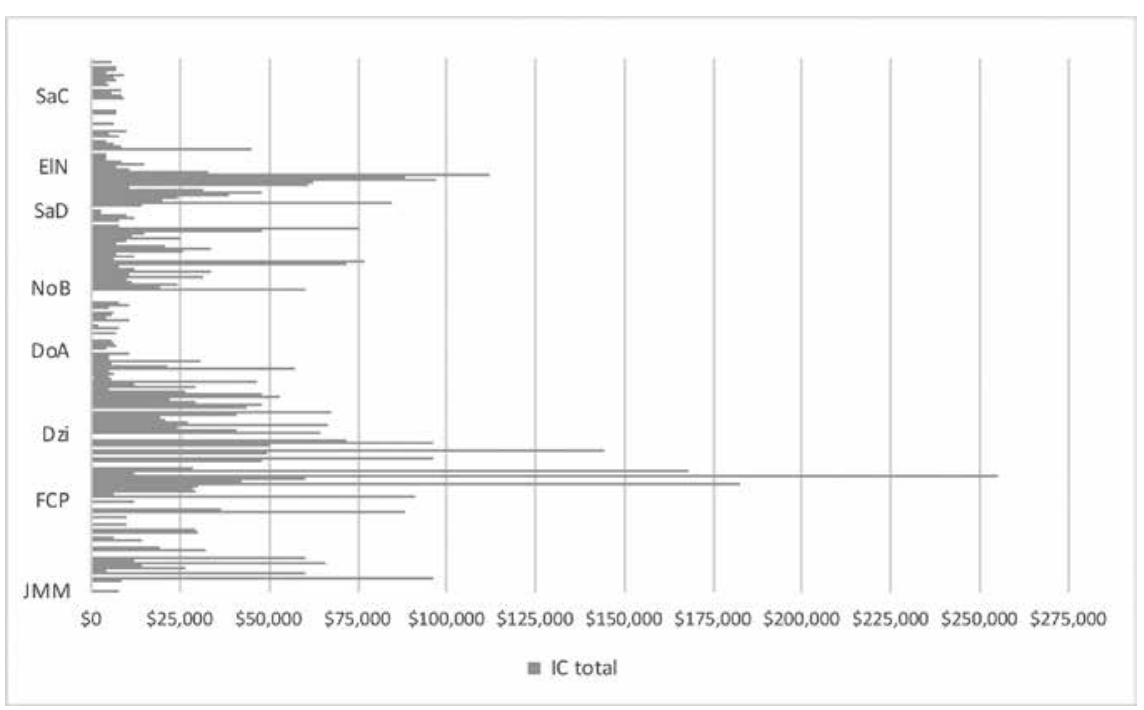

Figura 11: Ingreso corriente de mujeres por localidad.

La figura 5, de la cabecera municipal José María Morelos", muestra que el 64.47\% de las mujeres no tienen ingreso alguno, 3.94\% de las mujeres que tienen algún ingreso están por debajo de la línea de pobreza extrema de Banco Mundial, es decir, 68.41\% perteneces a esta categoría. Ahora bien, nueve mujeres (11.84\%) superan la línea de pobreza extrema, pero no la de bienestar mínimo que establece el CONEVAL, o sea, 80.26\% de las mujeres de esta localidad viven con ingresos por debajo del bienestar mínimo. Solo se registran cuatro casos que superan la línea de bienestar mínimo, pero no la de bienestar, lo que arroja un total de $84.21 \%$ en situación de algún tipo de pobreza.

La figura 6, de la otra cabecera municipal, Felipe Carrillo Puerto", da cuenta de que el $48.26 \%$ de las mujeres no tienen ingreso alguno, $1.78 \%$ de las mujeres que tienen algún ingreso están por debajo de la línea de pobreza extrema de Banco Mundial, es decir, $50.04 \%$ pertenecen a esta categoría. Ahora bien, cinco mujeres $(8.92 \%)$ superan la línea de pobreza extrema, pero no la de bienestar mínimo que establece el CONEVAL (año), o sea, $57.14 \%$ de las mujeres de esta localidad viven con ingresos por debajo del bienestar mínimo. Hay cuatro casos de mujeres que superan la línea de bienestar mínimo (\$32.64 diarios) por solo 1 peso. Aunadas a ellas, existen también otra mujer con ingreso por encima de dicha línea, lo que acumula un total de $8.92 \%$ de casos por encima de la línea de bienestar mínimo, pero por debajo de la de bienestar, lo que arroja un total de $66.07 \%$ en situación de algún tipo de pobreza. 


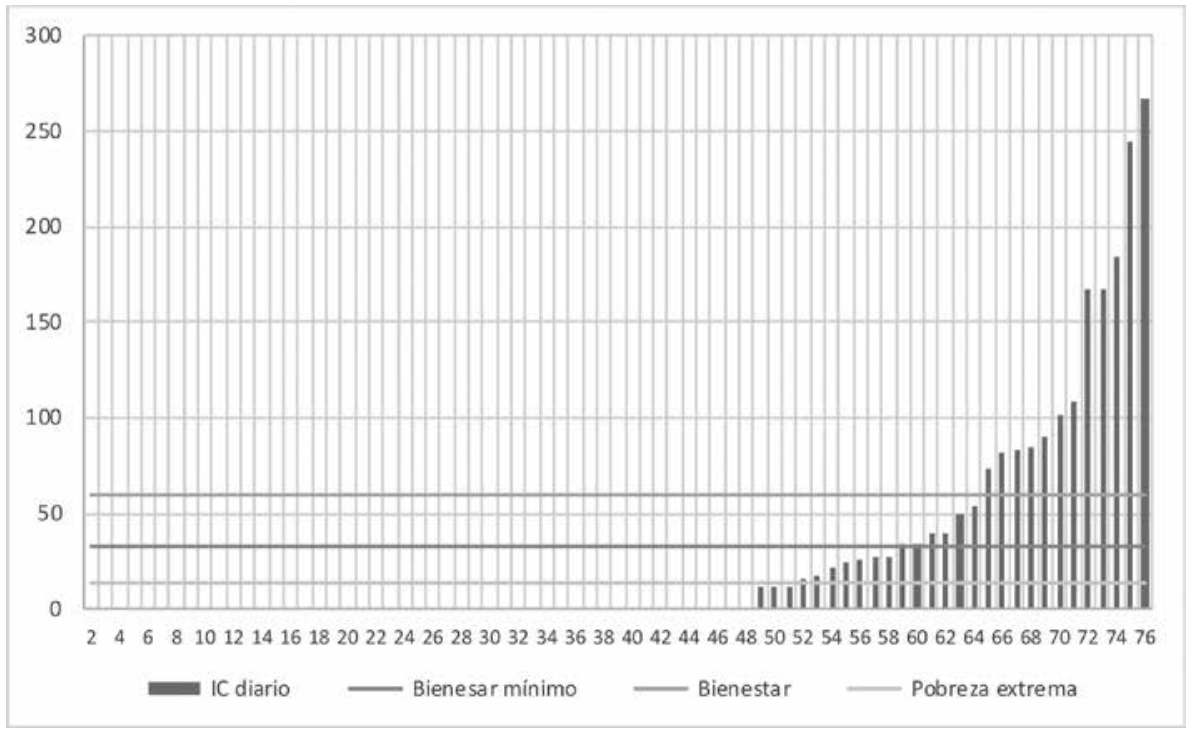

Figura 12: Líneas de pobreza para José María Morelos, Quintana Roo (2015)

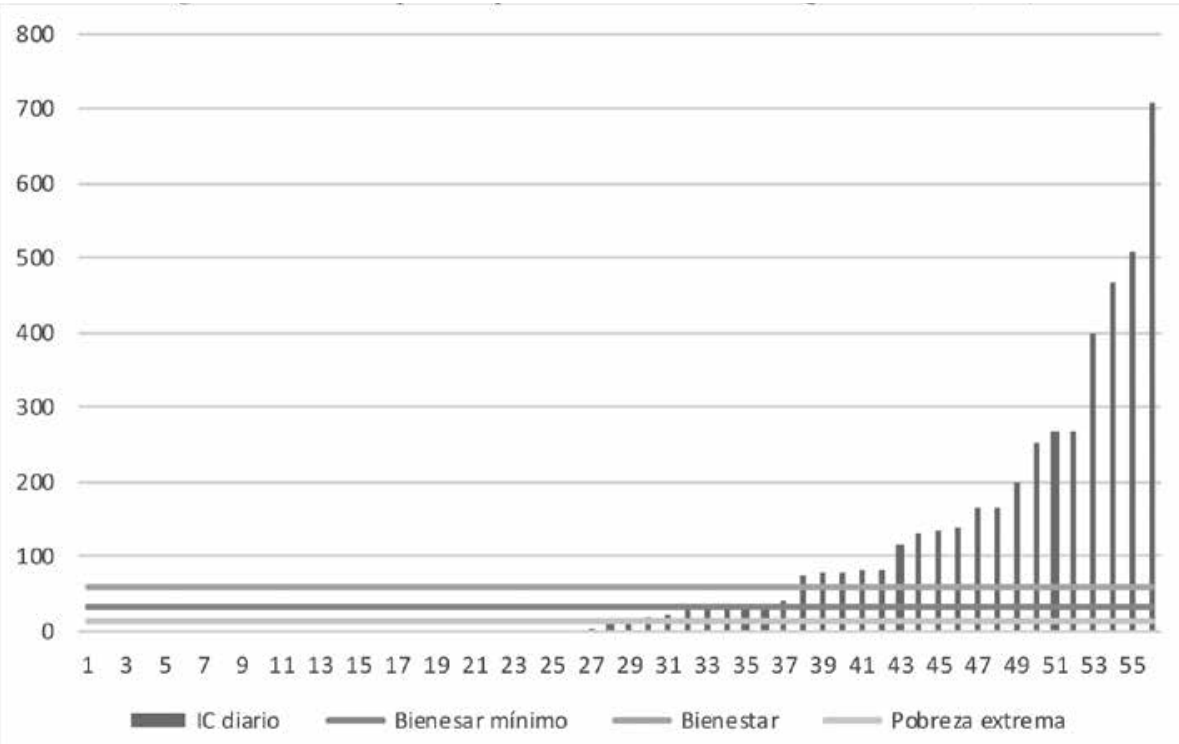

Figura 13: Líneas de pobreza para Felipe Carrillo Puerto, Quintana Roo (2015)

La figura 7, de la alcaldía Dziuché, muestra que el 18.57\% de las mujeres no tienen ingreso alguno (y dos casos tienen ingres diario de menos de un peso), $24.28 \%$ de las mujeres que tienen algún ingreso están por debajo de la línea de pobreza extrema de Banco Mundial, es decir, 42.85\% pertenece a esta categoría. Ahora bien, 21.42\% superan la línea de pobreza extrema, pero no la de bienestar mínimo que establece el 
CONEVAL (S/F), o sea, $64.28 \%$ de las mujeres de esta localidad viven con ingresos por debajo del bienestar mínimo. Sólo se registran cuatro casos que superan la línea de bienestar mínimo, pero no la de bienestar, lo que arroja un total de $70 \%$ en situación de algún tipo de pobreza.

La figura 8, perteneciente a Dos Aguadas, muestra que el 55.76\% de las mujeres de la muestra tienen un IC igual a cero lo que automáticamente las coloca por debajo de la línea de pobreza extrema. El 15.38\% reporta ingresos por debajo de la línea de pobreza extrema, lo que deriva en que sean en total $70 \%$ del total de mujeres las que viven con menos de $\$ 1.25$ dólares americanos al día. Ahora bien, el resto de las mujeres tiene un ingreso que no rebasa la línea de bienestar mínimo que CONEVAL (año) establece, es decir, para el gobierno mexicano, el 100\% de las mujeres de la muestra en Dos Aguadas viven en condiciones de pobreza extrema.

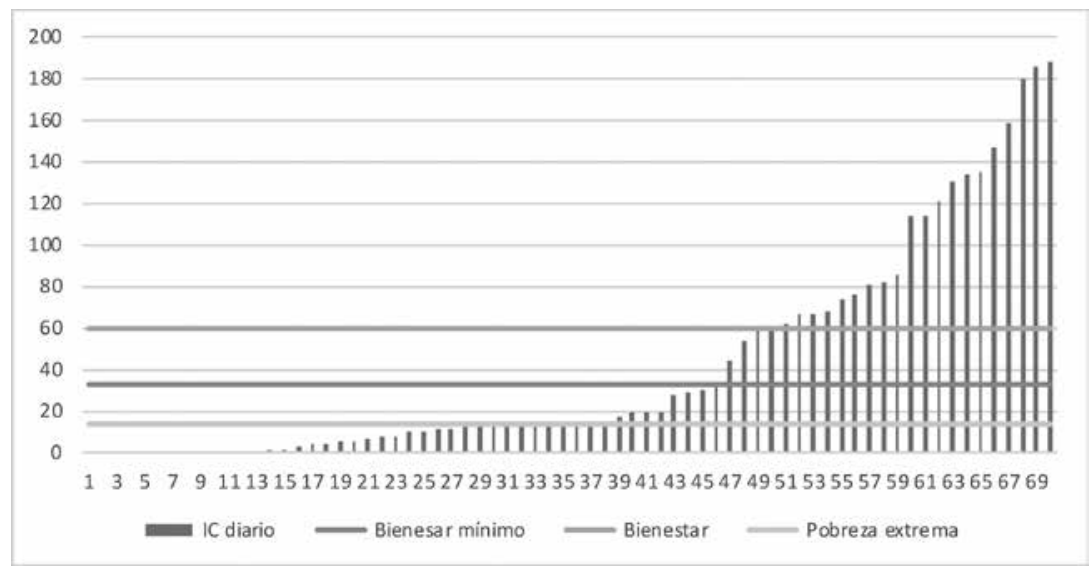

Figura 14: Líneas de pobreza para Dzluché, Quintana Roo (2015)

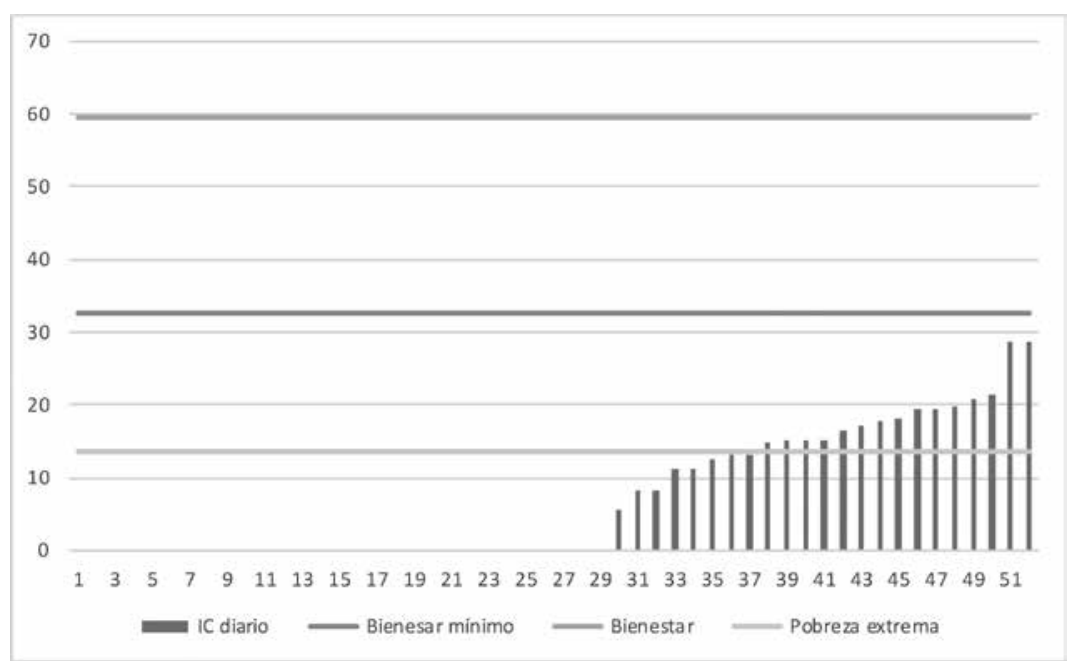

Figura 15: Líneas de pobreza para Dos Aguadas, Quintana Roo (2015) 
La figura 9, Noh Bec, muestra que el $\mathbf{2 6 . 1 5 \%}$ de las mujeres no tienen ingreso alguno. Cinco mujeres, es decir 7.69\% que tienen algún ingreso están por debajo de la línea de pobreza extrema de Banco Mundial, o sea, 33.84\% pertenece a esta categoría. Ahora bien, $38.46 \%$ superan la línea de pobreza extrema, pero no la de bienestar minimo que establece el CONEVAL (año), o sea, $72.30 \%$ de las mujeres de esta localidad viven con ingresos por debajo del bienestar mínimo. Solo se registran seis casos que superan la línea de bienestar mínimo, pero no la de bienestar, lo que arroja un total de $81.53 \%$ en situación de algún tipo de pobreza.

La figura 10, de San Diego, muestra que el 27.02\% de las mujeres no tienen ingreso alguno. Tres mujeres, es decir $8.10 \%$ que tienen algún ingreso están por debajo de la línea de pobreza extrema de Banco Mundial, o sea, 35.12\% pertenece a esta categoría. Ahora bien, $18.91 \%$ superan la línea de pobreza extrema, pero no la de bienestar mínimo que establece el CONEVAL, o sea, 54.05\% de las mujeres de esta localidad viven con ingresos por debajo del bienestar mínimo. Solo se registran tres casos que superan la línea de bienestar mínimo, pero no la de bienestar, lo que arroja un total de $62.16 \%$ en situación de algún tipo de pobreza.

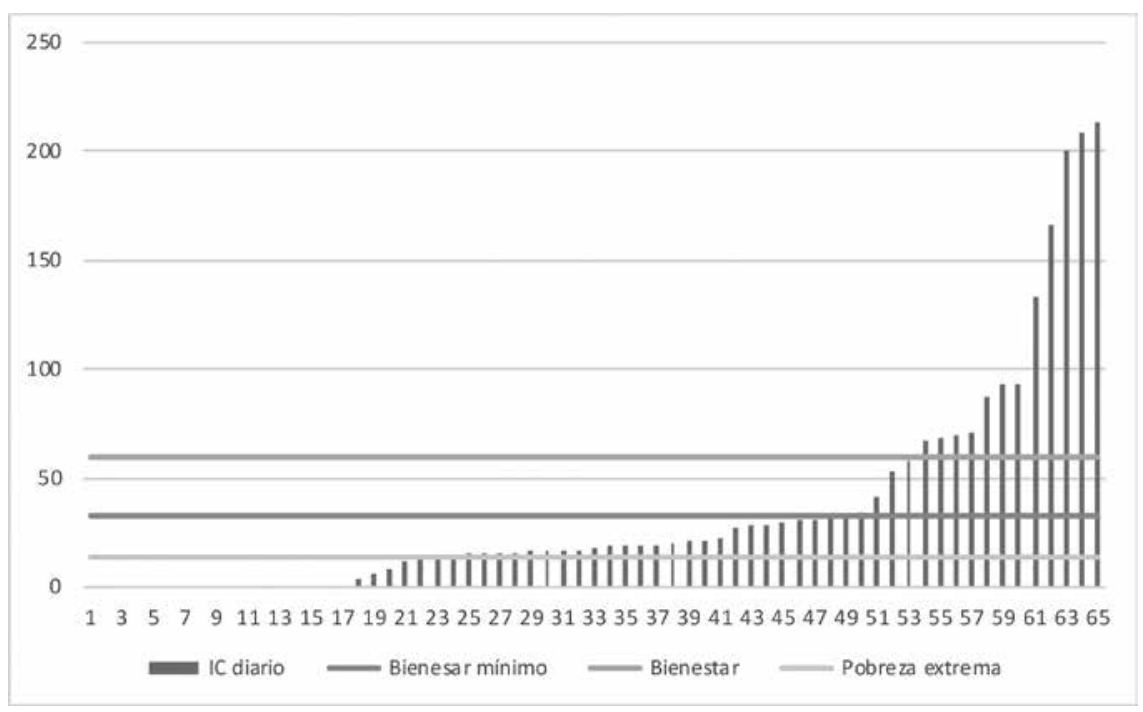

Figura 16: Líneas de pobreza para Noh Bec, Quintana Roo (2015) 


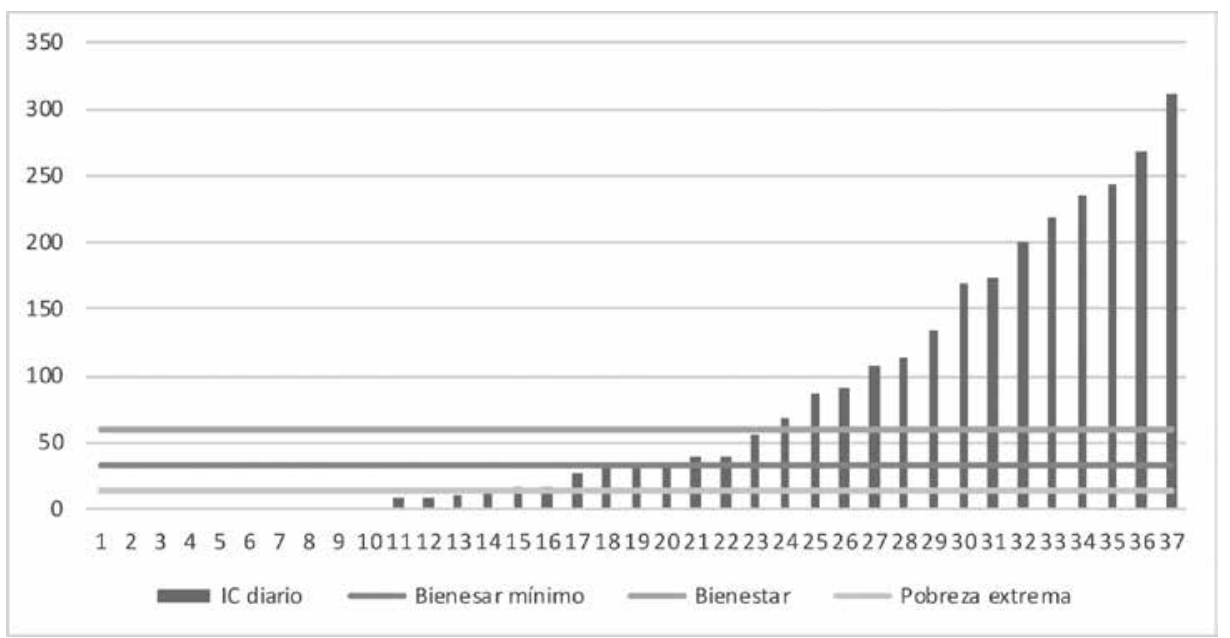

Figura 17: Líneas de pobreza para San Diego, Quintana Roo (2015)

La figura 11, de San Carlos, la localidad más pobre de la muestra y donde están los hogares con el menor IC, muestra que el $100 \%$ de la población femenina está por debajo de la línea de bienestar mínimo. El 42.42\% carece de algún ingreso, mientras que aquellas mujeres que registran algún ingreso, tres (9.09\%) están por debajo de la línea de pobreza extrema. Y el resto, el $48.48 \%$ tiene ingresos por encima de la línea de pobreza, pero por debajo de la línea de bienestar. De hecho, el ingreso más alto registrado per cápita es de $\$ 25$ pesos mexicanos diarios.

La figura 12, de El Naranjal, muestra que el 61.01\% de las mujeres no tienen ingreso alguno. Seis mujeres, es decir 10.16\% que tienen algún ingreso están por debajo de la línea de pobreza extrema de Banco Mundial, o sea, $71.17 \%$ pertenece a esta categoría. Ahora bien, $26.78 \%$ superan la línea de pobreza extrema, pero no la de bienestar mínimo que establece el CONEVAL, o sea, $96.61 \%$ de las mujeres de esta localidad viven con ingresos por debajo del bienestar mínimo. Solo se registran un caso que supera la línea de bienestar mínimo, pero no la de bienestar, lo que arroja un total de $98.30 \%$ en situación de algún tipo de pobreza 


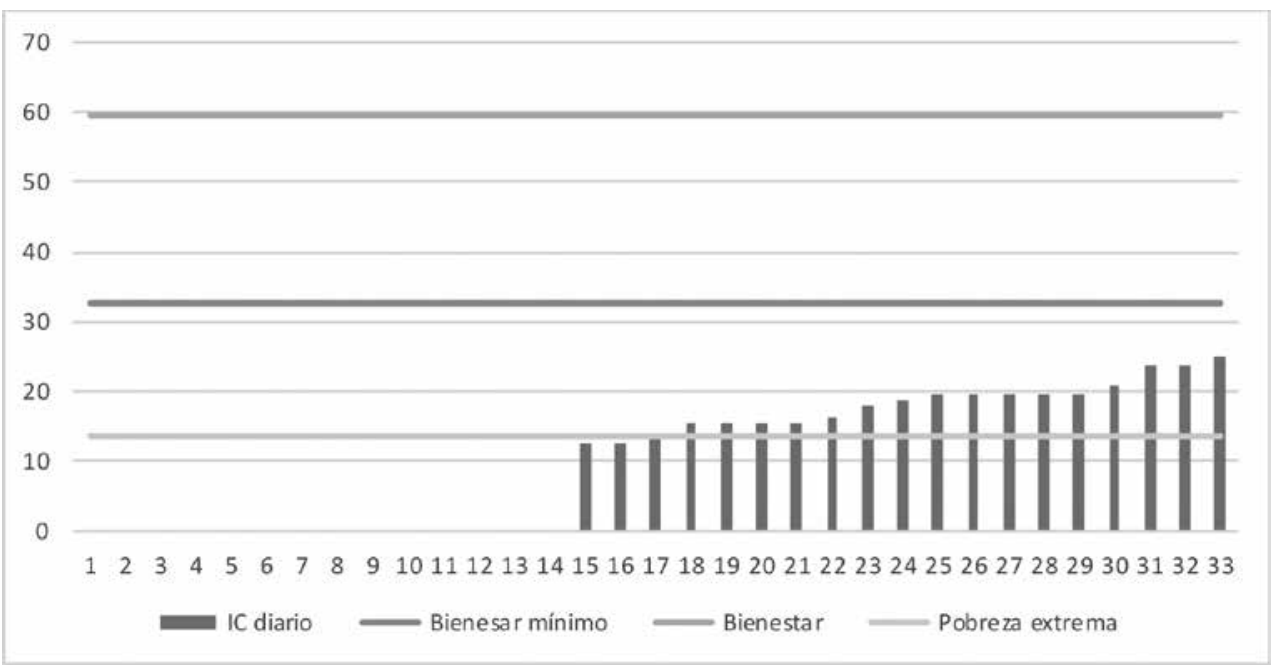

Figura 18: Líneas de pobreza para San Carlos, Quintana Roo (2015)

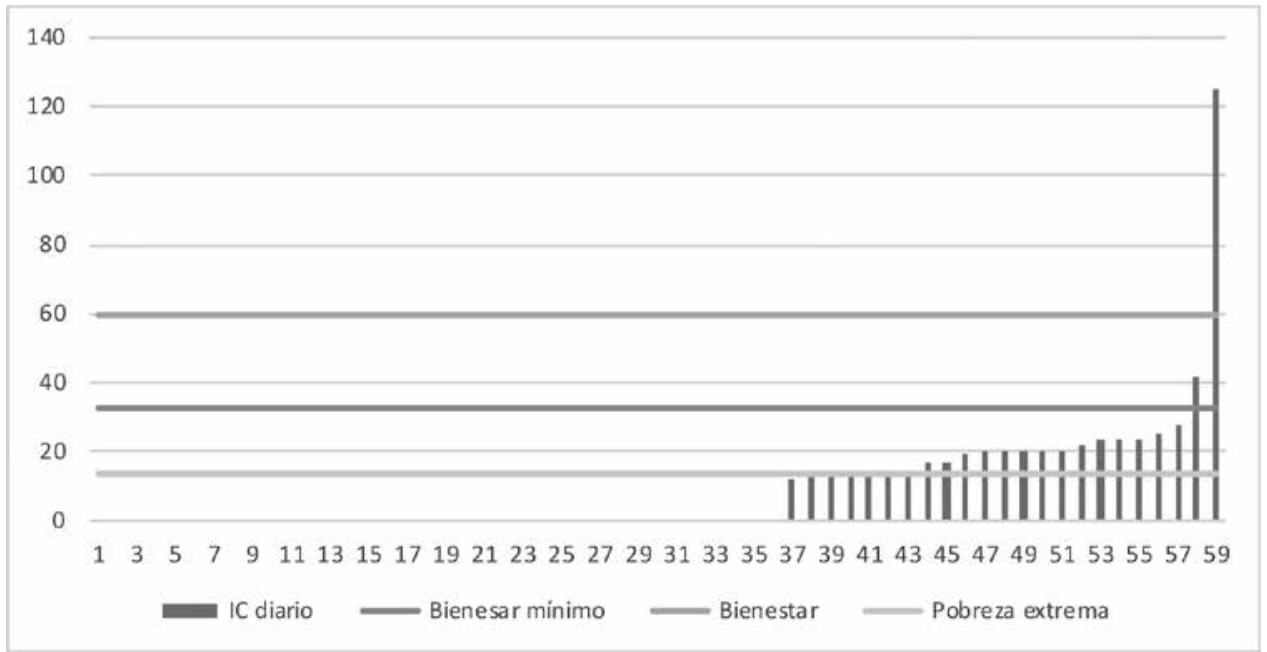

Figura 19: Líneas de pobreza para el Naranjal, Quintana Roo (2015)

\section{Conclusiones}

Lo que se observó durante el trabajo de campo, lo lacerante y bárbaro de la pobreza en que viven las mujeres, no hay modo de explicarlo al hipotético lector más que a través de este trabajo. Tal pareciera que en México ser indígena no es el último escalón de la 
desigualdad, ser mujer indígena sí que lo es. Aun cuando las políticas mexicanas de combate a la pobreza se han enfocado y tienen como objetivo el empoderamiento de la mujer, estas han resultado insuficientes y están lejos de terminar con la precaria situación de las mujeres indígenas. En los datos arriba presentados en gráfica está esbozado el IC, mismo que considera en su estructura a las transferencias monetarias condicionadas, que son los apoyos monetarios gubernamentales, mismos que en su mayoría se entregan a mujeres y que nos hacen reflexionar sobre lo efímero que resultan los montos otorgados cuando se comparan contra los umbrales mínimos de bienestar que existen y que se utilizaron en este trabajo,

Quienes participamos en este trabajo, estudiantes que colaboraron en campo, colegas que revisaron y aportaron ideas, creemos en la urgencia de rediseñar las políticas de combate a la pobreza por otras donde realmente exista alivio de este fenómeno que como se explicó con anterioridad lacera más a las mujeres, especialmente a las indígenas, pues en su contexto, el trabajo que aportan al hogar es incuantificable en términos monetarios, lo que les deja en vil dependencia de las políticas que los gobiernos implementen. Asimismo urge que los tomadores de decisiones públicas conozcan datos fríos pero reales del poder adquisitivo de la población, pues como se evidenció en este texto la pobreza extrema, esa de menos de un dólar americano al día, no sólo está presente en el África subsahariana, o en islas de Caribe, esa pobreza extrema existe en uno de los estados con Índice de Desarrollo Humano más alto en México (o.754, mientras que el de México como país es 0.756 de acuerdo al último informe de la $\mathrm{ONU}$ ), que dicho sea de paso pertenece al grupo de veinte países con economías más dinámicas, G-20.

\section{Lista de referencias}

Banco Mundial (1992). La medición de la pobreza. Comercio exterior, 42 (4), 323-325.

Banco Mundial. (2001). Informe sobre el desarrollo mundial 2000/2001: Lucha contra la pobreza. Banco Mundial.

Banco Mundial. (2004). La pobreza en México: una evaluación de las condiciones, las tendencias y la estrategia del Gobierno. México, Banco Mundial.

Banco Mundial (2013). Informe anual 2013. Instituto de Relaciones Internacionales (IRI).

Bravo, R. (1998). Pobreza y desigualdad de género. Una propuesta para el diseño de indicadores, Serie de Documentos de Trabajo. Santiago de Chile, SERNAM

Bravo, R. (2004). Las metas del Milenio y la igualdad e género: el caso de Perú. Nueva York, ONU. 
CEPAL (1990). Latin America and the Caribbean: options to reduce the debt burden. Santiago de Chile, CEPAL

CEPAL (1996). The economic experience of the last fifteen years: Latin America and the Caribbean, 1980-1995, México, CEPAL.

CEPAL (2003). Hacia el objetivo del milenio de reducir la pobreza en América Latina y el Caribe. Santiago de Chile, CEPAL.

CEPAL, N. (2004). Entender la pobreza desde la perspectiva de género. CEPAL.

CEPAL (2014). Prospectiva y politica pública para el cambio estructural en América Latina y el Caribe, Santiago de Chile, CEPAL.

CEPAL (2016). "Objetivos de desarrollo del milenio en América Latina y el Caribe", disponible en https://www.cepal.org/cgi-bin/getprod.asp?xml=/MDG/noticias/ paginas/1/40211/P40211.xml\&xsl=/MDG/tpl/p18f.xsl\&base=/MDG/tpl/topbottom.xsl

CONEVAL (2010). Metodología para la medición multidimensional de la pobreza en México, México. CONEVAL.

CONEVAL (2014). Evolución de las líneas de bienestar y de la canasta alimentaria. Disponible en http://www.coneval.gob.mx/Medicion/MP/Paginas/Lineasde-bienestar-y-canasta-basica.aspx

Cornelius, W. y Myhre, D. (1998). The transformation of rural Mexico: reforming the ejido sector, Hispanic American Historical Review, 79 (3), pp. 559-561

Delgado, J. (1999). La nueva ruralidad en México. Investigaciones geográficas, (39), 82-93.

Escalante, F. (2015). Historia mínima del neoliberalismo, México, El Colegio de la Frontera Norte.

Esquivel, G. (2009). El mercado laboral rural en México: caracterización y agenda de investigación (No. E51-101). CEPAL.

Esquivel, G. (2015). Desigualdad extrema en México: concentración del poder económico y político. México, OXFAM.

Feres, J. C., y Mancero, X. (2001). Enfoques para la medición de la pobreza: breve revisión de la literatura. CEPAL. 
Gaxiola, A. y Castro, M. (2017). Desigualdad, Turismo y Bienestar: Análisis regional y por estados, según estrato urbano y rural. El Periplo Sustentable, (32).

Gillie, A. (2008). "The origin of the poverty line" The Economic History Review, 49 (4), $715-730$

INEGI (2013). Metodología de la Encuesta Nacional de Ingreso y Gasto de los Hogares de México, Disponible en http://www.inegi.org.mx/est/contenidos/espanol/ metodologias/encuestas/hogares/enigh1o_descripcion_bd.pdf

Jessoe, K., Manning, D., y Taylor, E. (2016). Climate Change and Labour Allocation in Rural Mexico: Evidence from Annual Fluctuations in Weather. The Economic Journal.

ONU (2000), Objetivos de desarrollo del milenio, Washington, ONU.

Ramírez, K. e Ibarra, A. (2015). Percepción local de los servicios ecológicos y de bienestar de la selva de la zona maya en Quintana Roo, México. Investigaciones Geográficas, Boletín del Instituto de Geografía, 2015(86), 67-81.

Sen, G. (1998). El empoderamiento como un enfoque a la pobreza. Género y pobreza. Nuevas dimensiones, (26).

Zepeda, E. (1994), "El gasto social en México: de la estabilización ortodoxa al neoliberalismo social" en Frontera Norte, Vol. 6, Núm 1e, Pp. 171-197. 